\title{
Camrelizumab Combined with FOLFOX4 Regimen as First-Line Therapy for Advanced Hepatocellular Carcinomas: A Sub-Cohort of a Multicenter Phase lb/ll Study
}

\author{
Hui Li, ${ }^{1, *}$ Shukui Qin, (DI ${ }^{1, *}$ \\ Ying Liu, (1) ${ }^{2}$ \\ Zhendong Chen, ${ }^{3}$ \\ Zhenggang Ren, ${ }^{4}$ \\ Jianping Xiong, ${ }^{5}$ \\ Zhiqiang Meng, ${ }^{6}$ \\ Xiao Zhang, ${ }^{7}$ Linna Wang, ${ }^{7}$ \\ Xiaojing Zhang, ${ }^{7}$ \\ Jianjun $\mathrm{Zou}^{7}$
}

'Department of Medical Oncology Center, Bayi Affiliated Hospital, Nanjing University of Chinese Medicine, Nanjing, People's Republic of China; ${ }^{2}$ Department of Oncology, Cancer Hospital of Henan Province, Zhengzhou, People's Republic of China; ${ }^{3}$ Department of Clinical Oncology, The Second Affiliated Hospital of Anhui Medical University, Hefei, People's Republic of China; ${ }^{4}$ Department of Clinical Oncology, Zhongshan Hospital, Fudan University, Shanghai, People's Republic of China; ${ }^{5}$ Department of Clinical Oncology, The First Affiliated Hospital of Nanchang University, Nanchang, People's Republic of China; ${ }^{6}$ Minimally Invasive Treatment Center, Fudan University Shanghai Cancer Center, Shanghai, People's Republic of China; ${ }^{7}$ Jiangsu Hengrui Medicine Co., Ltd, Shanghai,

People's Republic of China

*These authors contributed equally to this work

Correspondence: Shukui Qin

Department of Medical Oncology Center Bayi Affiliated Hospital, Nanjing University of Chinese Medicine, No. 34, 34 Biao, Yanggongjing Street, Nanjing, 210002,

People's Republic of China

$\mathrm{Tel}+86-25-8086454$ l

Email qinsk@csco.org.cn
Background: Immune checkpoint inhibitors and chemotherapy can synergistically increase efficacy in a variety of malignancies. We conducted this phase Ib/II study to assess the safety and efficacy of anti-PD-1 antibody camrelizumab in combination with FOLFOX4 for treatment-naive advanced hepatocellular carcinoma (aHCC).

Methods: This open-label, multicenter phase Ib/II study (NCT03092895) enrolled patients with aHCC and without prior systemic treatment for treatment with camrelizumab $(3 \mathrm{mg} / \mathrm{kg})$ and FOLFOX4 every two weeks. First, six patients were enrolled, followed by an additional 28 patients after dose-limiting toxicity cases were determined to be $<33 \%$ of patients. The primary endpoint was tolerability and safety of treatment.

Results: A total of 34 aHCC patients were enrolled and received study treatment. No doselimiting toxicity were observed in the first six patients enrolled. Twenty-nine (85.3\%) of the total 34 patients had grade $\geq 3$ treatment-related adverse events (TRAEs), with the most common ones being decreased neutrophil count $(55.9 \%)$ and decreased white blood cell count $(38.2 \%)$. No TRAEs-related deaths occurred. The objective response and disease control rate were $29.4 \%$ (95\% CI, 15.1-47.5) and 79.4\% (95\% CI, 62.1-91.3), respectively. The median duration of response, progression-free survival, and overall survival was 6.9 months (range, 3.3-11.5), 7.4 months (95\% CI, 3.9-9.2), and 11.7 months (95\% CI, 8.2-22.0), respectively.

Conclusion: Camrelizumab combined with FOLFOX4 for first-line treatment of patients with aHCC showed good safety and tolerability, with promising preliminary antitumor activity.

Keywords: PD-1 monoclonal antibody, camrelizumab, FOLFOX4 regimen, hepatocellular carcinoma, combination therapy

\section{Background}

Primary liver cancer (PLC; also referred to liver cancer) is a common malignant tumor of the digestive system, and $90 \%$ of cases are hepatocellular carcinomas (HCC). ${ }^{1}$ According to data published by GLOBOCAN, there were 85.4 million new cases of liver cancer and 782,000 deaths worldwide in 2018, with a mortality/ morbidity ratio of 0.9 . Moreover, the morbidity and mortality rate ranked sixth and second among malignant tumors, respectively. ${ }^{2}$ HCC are highly heterogeneous in terms of etiology, clinical manifestations and staging, treatment strategies and prognosis, and are often accompanied by underlying liver disease. The 5-year 
survival rate is approximately $15-19 \%$ in North America and only $12.1 \%$ in China. About $70-80 \%$ of patients are in the middle and advanced stages at the time of initial diagnosis, ${ }^{3,4}$ and are thus not amenable to surgery. If supportive care is provided only, the mean survival time of Asian patients (except in Japan) with advanced hepatocellular carcinoma (aHCC) is only about 4 months. ${ }^{5}$

In 2007, sorafenib overcame this therapeutic dilemma and paved the way for molecular targeted therapy, but its ORR was only $2-3 \%$ and median overall survival (OS) was prolonged by only $2-3$ months, which is far from satisfactory. ${ }^{5,6}$ According to the REFLECT study, on September 4, 2018, lenvatinib was approved as first-line treatment for aHCC indications. ${ }^{7}$ To overcome the limitations of targeting monotherapy, we published the results of the EACH study in $2013 ;{ }^{8}$ FOLFOX4 (infusional fluorouracil, leucovorin, and oxaliplatin) significantly prolonged median progression-free survival (PFS), objective response rate (ORR) and disease control rate (DCR) compared with doxorubicin, and mOS was also significantly prolonged in the Chinese cohort (5.9 months vs 4.3 months, $P=0.0281) .^{9}$ At present, sorafenib, lenvatinib, and FOLFOX4 become standard first-line treatment options of aHCC. ${ }^{10}$ Regorafenib, cabozantinib, and ramucirumab have been successively approved by the US Food and Drug Administration (FDA) for second-line treatment. ${ }^{11-13}$ But the disadvantages of systemic treatment remain unsolved, including short-term efficacy and limited survival benefit.

Immunotherapy plays an important role in treating solid tumors. Studies on immune checkpoint inhibitors (ICIs) mainly focused on blocking programmed cell death protein 1 (PD-1) or its ligand programmed deathligand 1 (PD-L1). The CheckMate 040 study showed that nivolumab achieved an ORR of $19.6 \%$ and a DCR of $64.5 \%$ in the treatment of HCC. ${ }^{14}$ Moreover, the 12 - and 18 -month overall survival (OS) rate was $60 \%$ and $44 \%$, respectively, for sorafenib-pretreated patients and $73 \%$ and $57 \%$, respectively, in sorafenib-naive patients, who could benefit regardless of hepatitis $\mathrm{B} / \mathrm{C}$ virus $(\mathrm{HBV} / \mathrm{HCV}$ ) infection and PD-L1 expression status. ${ }^{15}$ This study introduced a new era of HCC immunotherapy. On September 23, 2017, the US FDA conditionally approved nivolumab for second-line treatment of HCC after sorafenib resistance; On November 9, 2018, pembrolizumab was also approved based on the results of the KEYNOTE-224 study. ${ }^{16}$ Camrelizumab is an anti-PD-1 monoclonal antibody. The results of a prospective, open-label, multicenter
Phase II study of second-line treatment with camrelizumab for aHCC showed that the ORR was $14.7 \%$, DCR was $44.2 \%$, and the 6-month OS rate was $74.4 \% .{ }^{17}$ Based on this study, on March 18, 2020, camrelizumab was approved by the China National Medical Products Administration (NMPA) for second-line standard of care for aHCC. However, two large confirmatory Phase III clinical studies, CheckMate-459 and KEYNOTE-240, were successively published with negative results in $2019 .^{18,19}$ In addition, the ORR and survival benefit of this monotherapy were limited.

In recent years, several clinical studies have shown that ICIs combined with other strategies including antiangiogenic drugs, chemotherapy, and radiotherapy had synergistic or additive effects, as well as good safety without overlapping major adverse events. ${ }^{20}$ Combination therapy achieved good efficacy and survival benefit in several solid tumors such as advanced non-small-cell lung cancer (NSCLC), gastric cancer, and esophageal cancer. However, to date, there have been no report of immunotherapy combined with systemic chemotherapy for aHCC, and its feasibility needs to be actively explored.

Here, we report the results of a subgroup cohort of a phase $\mathrm{Ib} / \mathrm{II}$ clinical trial of a camrelizumab-containing combination therapy for primary liver cancer, in an effort to evaluate the safety, tolerability, and efficacy of camrelizumab combined with a FOLFOX4 regimen for treatment-naive aHCC.

\section{Methods}

\section{Study Design and Participants}

This is an open-label, multicenter phase Ib/II clinical trial (ClinicalTrials.gov NCT03092895) that was conducted at six hospitals (Supplementary Table S1). Main inclusion criteria were 18 to 70 years of age; patients with pathologically confirmed aHCC without prior systemic therapy (including targeted therapy or chemotherapy); patients not eligible for surgical or local therapy; at least one measurable lesion as defined by Response Evaluation Criteria in Solid Tumors (RECIST) version 1.1; Child-Pugh A or B liver function (scores $\leq 7$ ); Eastern Cooperative Oncology Group (ECOG) performance status of $0-1$; expected survival $\geq 12$ weeks; and normal major organ function evaluated by routine blood examination (hemoglobin $\geq 90 \mathrm{~g} / \mathrm{L}$, neutrophils count $\geq 1.5 \times 10^{9} / \mathrm{L}$, platelet count $\geq 80 \times 10^{9} / \mathrm{L}$ ), biochemical test (albumin $\geq 29 \mathrm{~g} / \mathrm{L}$, alanine aminotransferase and aspartate aminotransaminase $\leq 2.5 \times$ upper limit of normal [ULN], total 
bilirubin $\leq 1.5 \times \mathrm{ULN}$, creatinine $\leq 1.5 \times \mathrm{ULN})$, and prothrombin time-international normalized ratio (PT-INR) $\leq 2.3$ or PT exceeds the normal range of control for $\leq 6$ seconds. Patients complicated with hepatitis B virus (HBV) infection should have $\mathrm{HBV}$ DNA $<500 \mathrm{IU} / \mathrm{mL}$, and those with $\mathrm{HBV}$ or hepatitis $\mathrm{C}$ virus $(\mathrm{HCV})$ infection should receive standardized antiviral therapy. Written informed consent was voluntarily obtained from participating patients. The main exclusion criteria included previous treatment with anti-PD -1/PD-L1 immunotherapy; local treatment for the liver (including but not limited to surgery, radiation therapy, hepatic artery embolization, transarterial chemoembolization, hepatic artery perfusion, radiofrequency ablation, cryoablation, or percutaneous ethanol injection) within 4 weeks before enrollment; active, known, or suspected autoimmune diseases; and pre-existing central nervous system metastases.

This study was conducted in strict compliance with Good Clinical Practice Guidelines, the Declaration of Helsinki, and the study protocol. The study protocol and amendments involved in this document were reviewed and approved by the Ethics Committee of each study site. The names of Ethics Committees of all study sites are provided in Supplementary Table S1.

\section{Procedures}

The treatment regimen consisted of camrelizumab (3 mg/ $\mathrm{kg}$, 20-60 min infusion) and FOLFOX4 (day 1: oxaliplatin [85 mg/m $\mathrm{m}^{2}, 2-\mathrm{h}$ infusion] plus leucovorin $\left[200 \mathrm{mg} / \mathrm{m}^{2}\right.$, 2-h infusion], followed by 5 -fluorouracil [400 $\mathrm{mg} / \mathrm{m}^{2}$, intravenous bolus; $600 \mathrm{mg} / \mathrm{m}^{2}, 22-\mathrm{h}$ infusion]; day 2 : leucovorin [200 mg/m $\mathrm{m}^{2}, 2-\mathrm{h}$ infusion], followed by 5 -fluorouracil $\left[400 \mathrm{mg} / \mathrm{m}^{2}\right.$, intravenous bolus; $600 \mathrm{mg} / \mathrm{m}^{2}, 22-\mathrm{h}$ infusion]). Chemotherapy drugs should be given at least 30 minutes after the end of camrelizumab. The regimen was repeated every 2 weeks in a 4 -week cycle.

The patients continued to receive the above treatment until progressive disease (PD), intolerable toxicity, withdrawal of informed consent, discontinuation of treatment at the discretion of the investigator, or end of the study. At the time of initial radiographic assessment of PD, combination therapy was continued with the patient's informed consent if continued use was still likely to be of clinical benefit, as per the judgment of the investigator. No dose adjustment of camrelizumab was possible during treatment and only dose interruptions, up to a maximum of 6 weeks, were allowed. FOLFOX4 treatment should be suspended if grade $\geq 3-4$ hematologic or non-hematologic toxicities (except for hair loss, local symptoms, and sensory nerve) occurred; when these toxicities returned to grade $\leq 1$, dose of oxaliplatin should be reduced to $65 \mathrm{mg} / \mathrm{m}^{2}, 5$-fluorouracil of infusion be reduced to $300 \mathrm{mg} / \mathrm{m}^{2} \times 2$ days, and 5 -fluorouracil of intravenous bolus be reduced to $500 \mathrm{mg} / \mathrm{m}^{2} \times 2$ days. If grade $\geq 2$ cardiac toxicity occurred, FOLFOX4 treatment should also be suspended; when it returned to grade $\leq 1$, oxaliplatin should be reduced to $65 \mathrm{mg} / \mathrm{m}^{2}$ and 5-fluorouracil should be discontinued. If grade $\geq 3$ allergy occurred, FOLFOX4 treatment should be permanently discontinued. After dose reduction, a dose increase was not permitted during subsequent treatment; if toxicities of grade $\geq 3$ recurred after dose reduction of chemotherapeutic drugs, chemotherapy must be discontinued.

Adverse events (AEs) were assessed from the time of signing the informed consent form to 90 days after dosing; they were coded using MedDRA v20.0 and graded according to National Cancer Institute Common Terminology Criteria for Adverse Events (NCI CTCAE) v4.03. Tumor imaging assessments were performed every 8 weeks $( \pm 7$ days $)$ until PD or if the patient started another anticancer therapy. Tumor response was evaluated according to RECIST v1.1. If the response assessment was complete response (CR) or partial response (PR), radiographic confirmation was required 4 weeks after the first response.

\section{Outcomes}

The primary endpoint was the safety and tolerability of camrelizumab in combination with FOLFOX4. Secondary endpoints included ORR, DCR, duration of response (DoR), PFS, and OS. ORR was defined as the proportion of patients with CR or PR, and DCR was defined as the proportion of patients with $\mathrm{CR}, \mathrm{PR}$ or stable disease (SD). DoR was defined as the time from the first radiographic assessment of a tumor lesion as CR or PR to the first assessment of PD or death from any cause. PFS was defined as the time from beginning of treatment to the date of tumor progression or death. OS was defined as the time from beginning of treatment to the date of death from any cause.

\section{Statistical Analysis}

We determined sample sizes for this cohort on the basis of observed toxicities, not statistical considerations. Firstly, six patients were planned to enrolled to assess the dose-limiting toxicity (DLT) within 28 days after treatment. If the proportion of patients with DLT was < 
$33 \%$, we would further enroll up to 30 patients. All patients who received at least one dose of the study drug and had post-treatment safety evaluation data were included in the safety analysis set (SS). All patients who received at least one dose of study drug were included in the full analysis set (FAS). Safety analyses were based on the SS and efficacy analyses were based on the FAS. The Kaplan-Meier method was used to estimate the median time to OS, PFS, and DoR, and the Brookmeyer and Crowley method was used to estimate their bilateral 95\% confidence intervals (CIs). ORR and DCR were calculated and the Clopper-Pearson method was used to calculate the $95 \%$ CIs. All statistical analyses were performed using the statistical analysis software $\mathrm{SAS}^{\circledR}$ v9.4 (SAS Institute, Cary, NC, US).

\section{Results}

\section{Patient Baseline Characteristics}

From May 17, 2017 to August 19, 2018, a total of 34 patients with aHCC were enrolled and received the combination treatment of camrelizumab and FOLFOX4. All patients were included in the SS and FAS (Figure 1). As of July 15, 2019, the median follow-up time was 11.5 months (range, 2.7-22.4). The median duration of drug exposure was 7.4 months (range, 1.8-18.5) for camrelizumab and 4.2 months (range, 1.8-9.2) for FOLFOX4. As of data cutoff, $31(91.2 \%)$ patients discontinued treatment and 3 $(8.8 \%)$ remained on treatment. Reasons for treatment discontinuation included disease progression (23 patients, 67.6\%), consent withdrawal (4 patients, $11.8 \%$ ), adverse event (1 patient, 2.9\%), and others (3 patients, 8.8\%). After disease progression, 12 patients (35.3\%) received subsequent anti-tumor therapy, including targeted therapy (9 patients, 26.5\%), chemotherapy ( 1 patient, $2.9 \%$ ), radiotherapy ( 2 patients, $5.9 \%$ ), surgery (1 patient, $2.9 \%$ ), and other therapies (3 patients, $8.8 \%$ ).

Baseline characteristics are presented in Table 1. The patients had a median age of 52 years; 79.4\% were infected with chronic HBV; $85.3 \%$ were at Barcelona Clinic Liver Cancer (BCLC) stage C; $79.4 \%$ had extrahepatic metastases; and $79.4 \%$ had an ECOG score of 1 . All patients were treatment-naive aHCC.

\section{Safety and Tolerability}

The originally enrolled six patients exhibited no DLT. Out of all 34 patients, all patients experienced

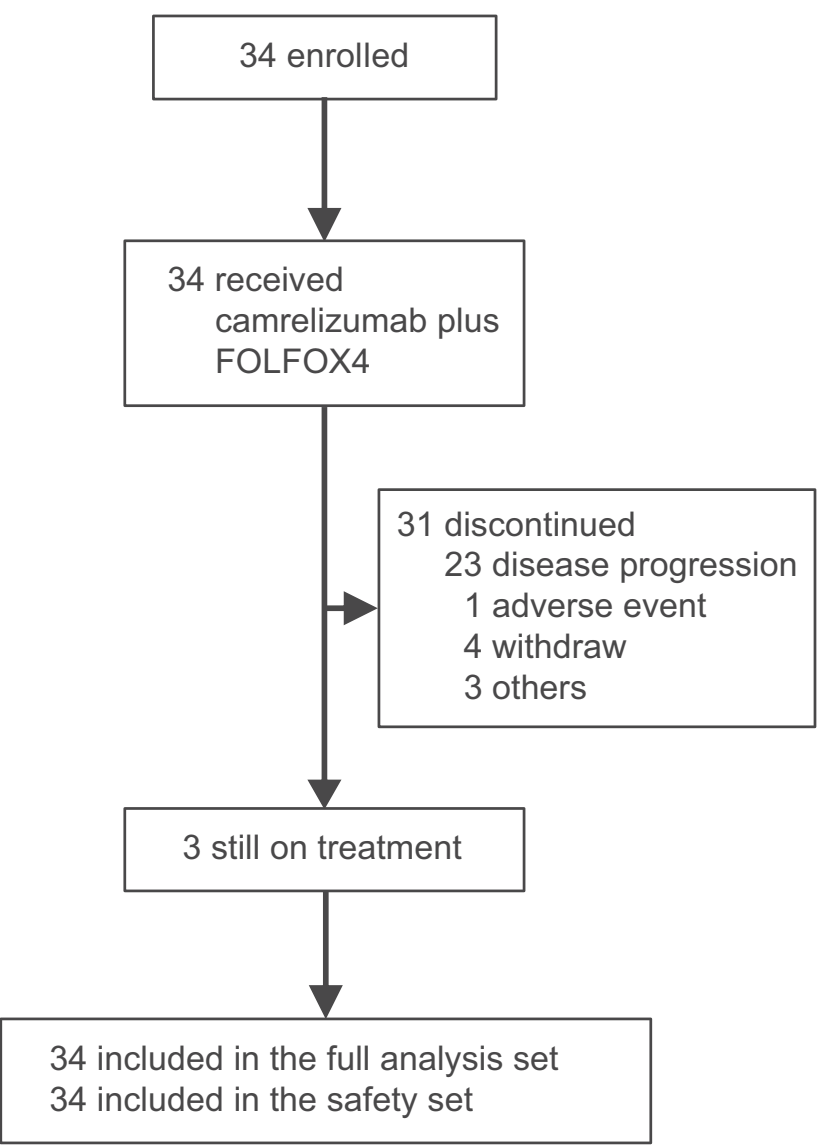

Figure I Trial profile.

treatment-related adverse events (TRAEs; Table 2). TRAEs with an incidence of $\geq 50 \%$ included decreased white blood cell count (28 patients, $82.4 \%$ ), decreased neutrophil count (27 patients, $79.4 \%$ ), decreased platelet count (26 patients, 76.5\%), and reactive cutaneous capillary endothelial proliferation (RCCEP; 25 patients, $73.5 \%$ ). The incidence of grade $\geq 3$ TRAEs were reported in $85.3 \%$ (29 patients) of patients, with the most common ones being decreased neutrophil count (19 patients, 55.9\%), decreased white blood cell count (13 patients, 38.2\%), and decreased platelet count (7 patients, 20.6\%). Fifteen patients (44.1\%) underwent treatment dose reductions or treatment interruptions due to TRAEs, but none of the TRAEs led to treatment discontinuation or death.

Five patients $(14.7 \%)$ had treatment-related serious adverse events (SAEs), including febrile neutropenia, bone marrow failure, hypersplenism, reactive capillary endothelial proliferation in nasal mucosal, myelosuppression, and duodenal ulcer (one patient each, 2.9\%). Of them, three $(8.8 \%)$ patients had drug-related SAEs of grade $\geq 3$. 
Table I Patient Demographics and Baseline Characteristics

\begin{tabular}{|c|c|}
\hline & $\begin{array}{l}\text { Camrelizumab Plus FOLFOX4 } \\
\qquad(\mathrm{N}=34)\end{array}$ \\
\hline Age, years, median (range) & $52(36-70)$ \\
\hline \multicolumn{2}{|l|}{ Gender } \\
\hline Male & 31 (91.2\%) \\
\hline Female & $3(8.8 \%)$ \\
\hline \multicolumn{2}{|l|}{ ECOG performance status } \\
\hline 0 & 7 (20.6\%) \\
\hline 1 & 27 (79.4\%) \\
\hline \multicolumn{2}{|l|}{ AFP } \\
\hline$<400 \mathrm{ng} / \mathrm{mL}$ & $13(38.2 \%)$ \\
\hline$\geq 400 \mathrm{ng} / \mathrm{mL}$ & 21 (6I.8\%) \\
\hline \multicolumn{2}{|l|}{$\mathrm{BCLC}$ stage } \\
\hline B & $4(11.8 \%)$ \\
\hline C & $29(85.3 \%)$ \\
\hline Unknown & I (2.9\%) \\
\hline \multicolumn{2}{|l|}{ Extrahepatic spread } \\
\hline Yes & 27 (79.4\%) \\
\hline No & 7 (20.6\%) \\
\hline \multicolumn{2}{|l|}{ Portal vein invasion } \\
\hline Yes & II (32.4\%) \\
\hline No & $23(67.6 \%)$ \\
\hline \multicolumn{2}{|l|}{ HBV infection } \\
\hline Yes & 27 (79.4\%) \\
\hline No & 7 (20.6\%) \\
\hline \multicolumn{2}{|l|}{ Child-Pugh class } \\
\hline$A(5-6)$ & $33(97.1 \%)$ \\
\hline B (7) & I (2.9\%) \\
\hline
\end{tabular}

Note: Data are N (\%), unless otherwise specified.

Abbreviations: ECOG, Eastern Cooperative Oncology Group; AFP, alphafetoprotein; BCLC, Barcelona Clinic Liver Cancer; HBV, hepatitis B virus.

RCCEP occurred in 25 patients (73.5\%), and three patients $(8.8 \%)$ presented with reactive capillary endothelial proliferation in non-cutaneous sites (oral gingiva, two patients; nasal mucosa, one patient). As of data cutoff date, five patients $(20.0 \%, 5 / 25)$ recovered from RCCEP, with a duration from last dose to remission of 6.3 weeks (range, -20.6-11.3). All the three patients $(100 \%, 3 / 3)$ with reactive capillary endothelial proliferation in non-cutaneous sites had recovered. We found that 25 patients who developed RCCEP had a numerically higher ORR than those who did not $(36.0 \%$ vs $11.1 \%, p=0.225$, Fisher exact test), but the difference between the two subgroups was not significant which mainly owing to the small sample size.

\section{Efficacy}

As of data cutoff, no patients achieved CR, 10 (29.4\%) patients had a PR, and $17(50.0 \%)$ had SD. The ORR was $29.4 \%$ (95\% CI, 15.1-47.5) and the DCR was $79.4 \%$ (95\% CI, 62.1-91.3). The DoR was 6.9 months (range, 3.3-11.5) and the median time to response was 2.0 months (range, 1.5-5.7). Best percentage changes in size of target lesions are presented in Figure 2A. Of the 10 patients with PR, $3(30.0 \%)$ were still in sustained response (Figure 2B), and the duration of response was 9.3, 9.5, and 11.0 months, respectively. The median PFS (mPFS) was 7.4 months (95\% CI, 3.9-9.2; Figure 3A). Twenty-one (61.8\%) patients died while 13 (38.2\%) were still alive at follow-up, with a median OS (mOS) of 11.7 months (95\% CI, 8.2-22.0; Figure 3B). The 6- and 12month OS was 79.4\% (95\% CI, 61.6-89.6) and 50.0\% (95\% CI, 32.4-65.3), respectively.

\section{Discussion}

The liver is considered an immune-privileged organ, with a special immunosuppressive cell population. HBV- and HCV-related HCC exhibits immunogenicity, and the HBV infection rate in patients with $\mathrm{HCC}$ is as high as $77 \%$ especially in China. ${ }^{21}$ Novel immunotherapy represented by ICIs is becoming an important strategy for HCC treatment, and several studies have demonstrated its significant efficacy. ${ }^{14,16,17}$ However, CheckMate-459 and KEYNOTE-240 showed that nivolumab or pembrolizumab had improved ORR compared with sorafenib, ${ }^{18,19}$ but the primary study endpoint of OS did not reach a statistically significant difference.

For aHCC treatment, the ORR rate of ICI monotherapy is between $15 \%$ and $20 \%$, which remains unsatisfactory; therefore, increased attention has been given to exploring the immunotherapy-dominated "combination therapy mode", 20 such as immunotherapy in combination with immunotherapy, targeted therapy, systemic chemotherapy, anti-angiogenic drugs, or local treatment, etc., in an effort to significantly improve efficacy. Both KEYNOTE-524 and IMbrave-150 studies achieved positive results, ${ }^{22,23}$ reflecting the success of immunotherapy in combination with targeted drug or anti-angiogenic drug in the treatment of aHCC, greatly encouraging other combination therapy modalities. There have been no reports of immunotherapy in combination with systemic chemotherapy for $\mathrm{HCC}$ treatment, but this treatment paradigm has shown superior 
Table 2 Treatment-Related Adverse Events

\begin{tabular}{|c|c|c|c|c|}
\hline & \multicolumn{4}{|c|}{ Camrelizumab Plus FOLFOX4 ( $N=34)$} \\
\hline & \multirow[t]{2}{*}{ Any Grade } & \multicolumn{3}{|c|}{ Grade $\geq 3$} \\
\hline & & Total & Grade 3 & Grade 4 \\
\hline TRAE & $34(100 \%)$ & $29(85.3 \%)$ & $22(64.7 \%)$ & $7(20.6 \%)$ \\
\hline White blood cell count decreased & $28(82.4 \%)$ & $13(38.2 \%)$ & $13(38.2 \%)$ & 0 \\
\hline Neutrophil count decreased & 27 (79.4\%) & 19 (55.9\%) & $13(38.2 \%)$ & $6(17.6 \%)$ \\
\hline Platelet count decreased & $26(76.5 \%)$ & $7(20.6 \%)$ & $7(20.6 \%)$ & 0 \\
\hline RCCEP & $25(73.5 \%)$ & 0 & 0 & 0 \\
\hline Decreased appetite & $13(38.2 \%)$ & 0 & 0 & 0 \\
\hline Anemia & II (32.4\%) & $2(5.9 \%)$ & $2(5.9 \%)$ & 0 \\
\hline Aspartate aminotransferase increased & $9(26.5 \%)$ & I (2.9\%) & I (2.9\%) & 0 \\
\hline Alanine aminotransferase increased & $8(23.5 \%)$ & 0 & 0 & 0 \\
\hline Nausea & $8(23.5 \%)$ & I (2.9\%) & I (2.9\%) & 0 \\
\hline Hypoesthesia & $7(20.6 \%)$ & I (2.9\%) & I (2.9\%) & 0 \\
\hline Lipase increased & $6(17.6 \%)$ & $3(8.8 \%)$ & $2(5.9 \%)$ & I (2.9\%) \\
\hline Vomiting & $6(17.6 \%)$ & $2(5.9 \%)$ & $2(5.9 \%)$ & 0 \\
\hline Pyrexia & $6(17.6 \%)$ & 0 & 0 & 0 \\
\hline Asthenia & $6(17.6 \%)$ & I (2.9\%) & I (2.9\%) & 0 \\
\hline Hypersensitivity & $6(17.6 \%)$ & $2(5.9 \%)$ & $2(5.9 \%)$ & 0 \\
\hline Bilirubin conjugated increased & $5(14.7 \%)$ & I (2.9\%) & I (2.9\%) & 0 \\
\hline Rash & $5(14.7 \%)$ & 0 & 0 & 0 \\
\hline Gamma-glutamyltransferase increased & $4(11.8 \%)$ & $2(5.9 \%)$ & $2(5.9 \%)$ & 0 \\
\hline Amylase increased & $4(11.8 \%)$ & 0 & 0 & 0 \\
\hline Blood bilirubin increased & $4(11.8 \%)$ & 0 & 0 & 0 \\
\hline Proteinuria & $4(11.8 \%)$ & 0 & 0 & 0 \\
\hline
\end{tabular}

Note: Data are N (\%).

Abbreviations: TRAE, treatment-related adverse events; RCCEP, reactive cutaneous capillary endothelial proliferation. No grade 5 TRAE occurred. Any grade TRAEs occurring in at least $10 \%$ of patients are listed.

efficacy to monotherapy in a variety of other solid tumors, such as NSCLC and nasopharyngeal carcinoma. ${ }^{24-30}$

Systemic chemotherapy can inhibit and kill tumor cells, releasing a large number of $\mathrm{T}$ cell chemokines after exposure to antigens and leading to $\mathrm{T}$ cell accumulation around the tumor, which is then exploited by immunotherapy; therefore, this combination therapy can control tumor development, gaining time for immunotherapy to response and synergizing the treatment effects. ${ }^{31-33}$ Some chemotherapeutic drugs, represented by oxaliplatin, enhance the immune response of tumor cells by inducing immunogenic cell death (ICD), ${ }^{31}$ acting on cellular signal transducer and activator of transcription (STAT) protein signaling pathways, $^{34}$ and regulating the tumor microenvironment. ${ }^{35,36}$ The three key events that induce ICD are exposure to calreticulin and heat shock proteins, ATP leakage, and high mobility group box 1 (HMGB-1) release, all of which are indispensable. ${ }^{31}$ In addition, combination therapy may also alter the immune status by regulating the expression of immune checkpoints. ${ }^{37}$ A study employing animal models of colorectal cancer demonstrated a good synergistic effect of oxaliplatincontaining chemotherapy regimens in combination with ICIs. $^{38}$ Relevant clinical studies on the treatment of a variety of solid tumors, including gastric, colorectal, pancreatic, and esophageal cancers are currently underway.

This study is the first to explore the safety and efficacy of anti-PD-1 monoclonal antibody combined with FOLFOX4 systemic chemotherapy for patients with treatment-naive aHCC. The results showed that most TRAEs were well tolerated and manageable. The most common TRAEs were hematologic abnormalities and RCCEP; No deaths were caused by TRAEs. Our results are similar to the incidence of hematological toxicity of camrelizumab in combination with chemotherapy for other cancer varieties. $^{27,30}$ RCCEP was the most common TRAE of camrelizumab, with an incidence as high as $73.5 \%$ in this study, but all cases were grade 1-2 and were mainly distributed on the skin surface, which recovered spontaneously after drug withdrawal. Notably, this TRAE was positively correlated with $\mathrm{ORR},{ }^{39}$ and its specific 

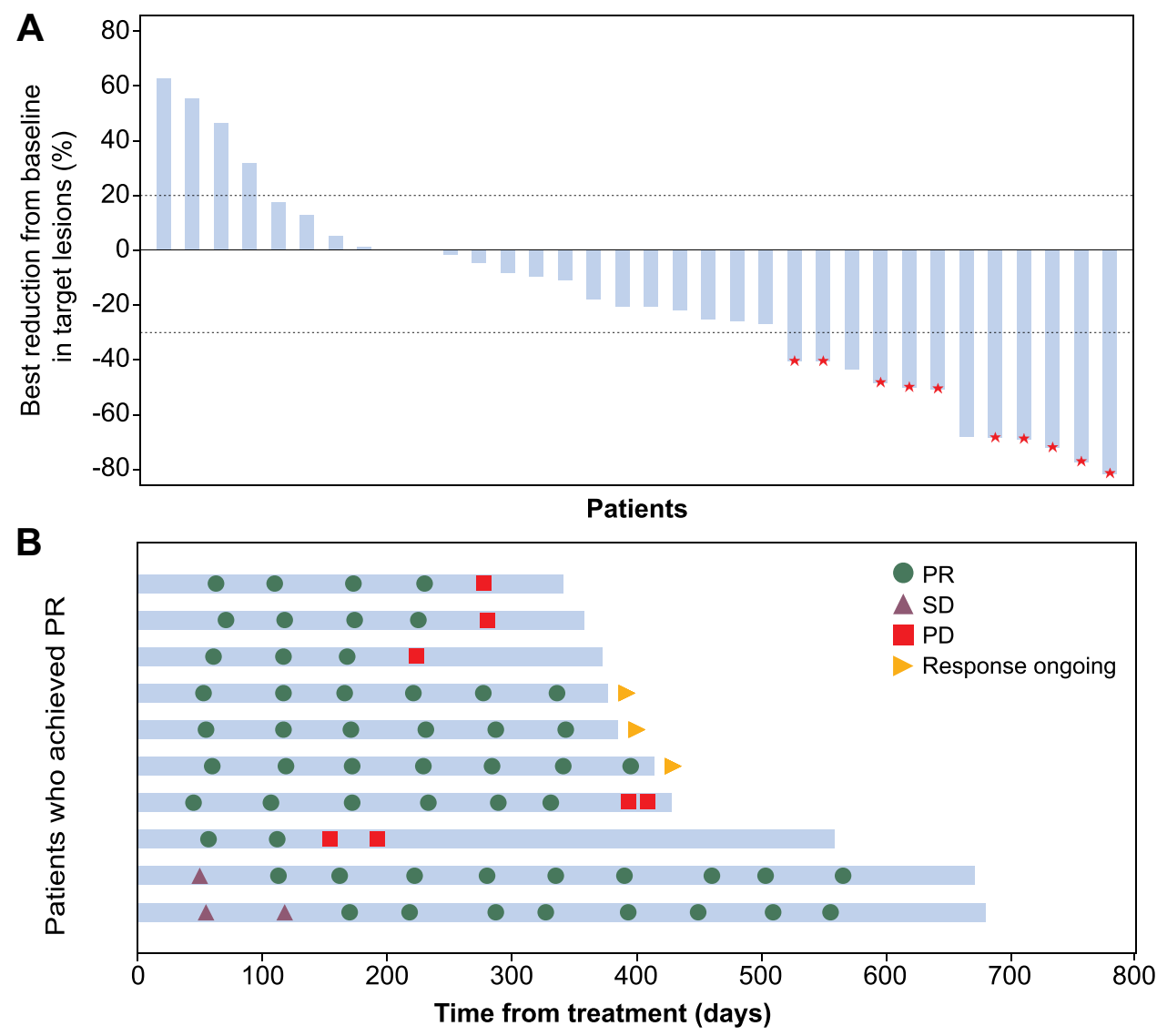

Figure 2 Tumor response after treatment. (A) Best changes from baseline in size of target lesions. Red star indicates confirmed response. (B) Time to response and duration of response.

mechanism and value in predicting efficacy require confirmation in further studies.

Compared with similar studies, ${ }^{24,25}$ patients in our study had complex conditions and worse general conditions at baseline (Supplementary Table S2). The majority of patients were complicated with HBV infection and extrahepatic metastasis, and the baseline tumor stage was late. Results showed that both ORR and DCR in our study were higher than those of nivolumab in the CheckMate 040 study (ORR, 19.6\%; DCR, 64.5\%) and pembrolizumab in the KEYNOTE-224 study (ORR, $17.3 \%$; DCR, 61.5\%), ${ }^{14,16}$ and also higher than the those of camrelizumab alone (ORR, 14.7\%; DCR, $44.2 \%$ ) or FOLFOX4 regimen (ORR, 8.2\%; DCR, $52.7 \%$ ) in the treatment of aHCC. ${ }^{8,17}$ Although crosstrial comparison should be interpreted with caution due to the different patient populations included, this study demonstrated the promising efficacy of camrelizumab plus FOLFOX4 in aHCC. Thus, the results are of great value and are encouraging.
There are, of course, some limitations associated with this study. The sample size included was small, and thus relevant conclusions need to be verified in studies with large sample sizes. Second, this study had no hypothesis testing and we did not assign a randomized control group. Third, comparison of the efficacy between patients with extrahepatic metastasis and without metastasis was lacking since bias would be introduced owing to the small sample size and the unbalanced patient number in the two subgroups. Nevertheless, we have already conducted a prospective randomized double-blind, parallelcontrolled, domestic multicenter phase III study to determine the efficacy and safety of camrelizumab plus FOLFOX4 vs placebo plus FOLFOX4 for the first-line treatment of aHCC (NCT03605706), which shows great promise.

In summary, this study showed that for aHCC, the toxicity of camrelizumab combined with the FOLFOX4 regimen was tolerable and the safety was controllable. Moreover, the excellent efficacy was preliminarily 

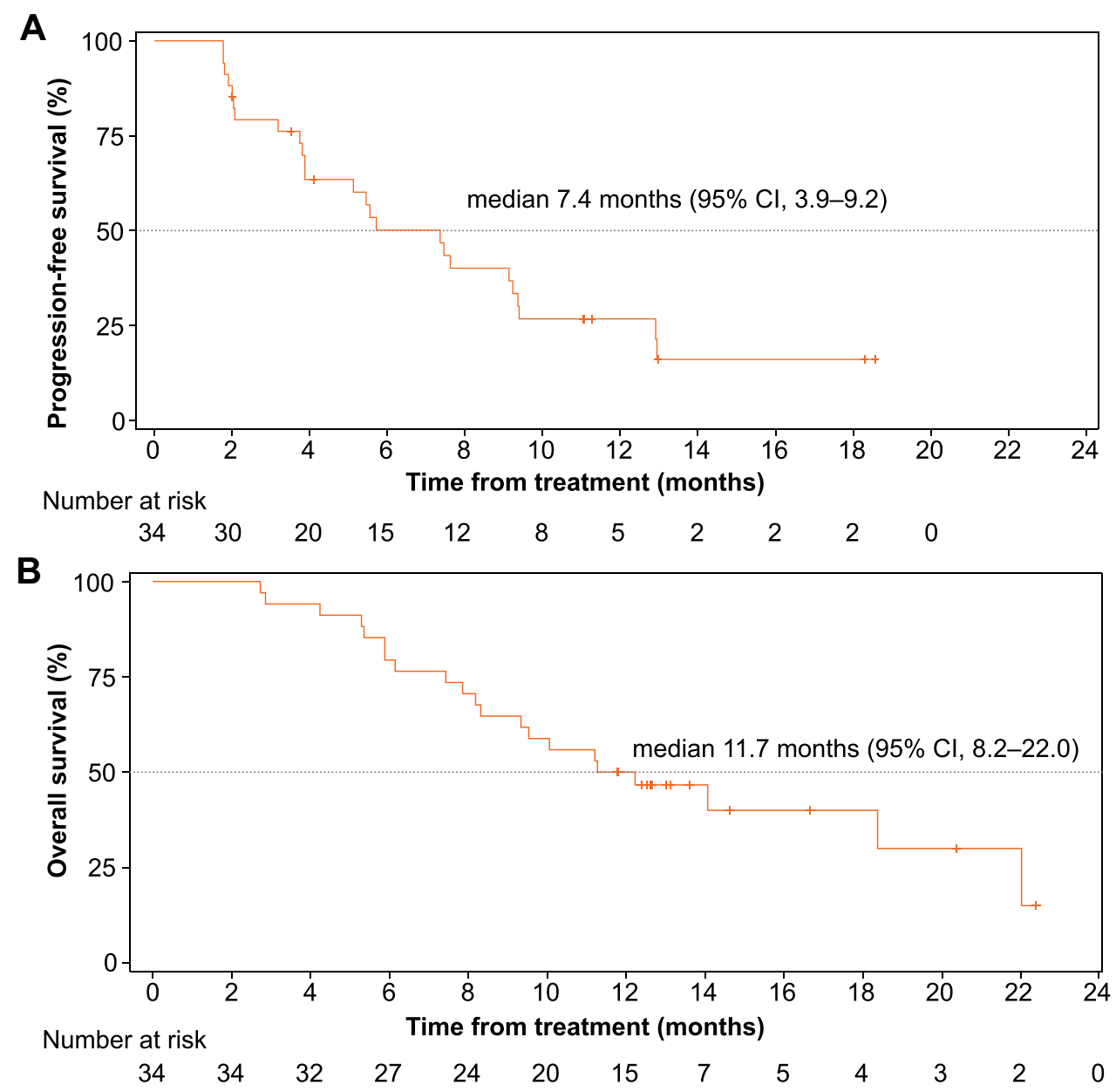

Figure 3 Kaplan-Meier estimates of progression-free survival (A) and overall survival (B)

demonstrated, providing an important foundation for further clinical studies.

\section{Data Sharing Statement}

The datasets used and/or analyzed during the current study are available from the corresponding author on reasonable request.

\section{Ethics Approval and Consent to Participate}

This study was conducted in strict compliance with Good Clinical Practice Guidelines, the Declaration of Helsinki, and the study protocol. The study protocol and amendments involved in this document were reviewed and approved by the Ethics Committees of each study site.

\section{Consent for Publication}

Not applicable.

\section{Acknowledgments}

The sponsor of this study is Jiangsu Hengrui Medicine Co., Ltd. My heartfelt thanks to all the patients and their families, as well as to all the researchers and medical staff involved in this study. Medical writing for this manuscript was provided by Tengfei Zhang, PhD (Medical Writer at Hengrui) according to Good Publication Practice Guidelines.

This study was presented in part at the American Society of Clinical Oncology (ASCO) Annual Meeting as a poster presentation on June 03, 2019. The poster's abstract was published in 'Poster Abstracts' in Journal of Clinical Oncology: https://ascopubs.org/doi/abs/10.1200/ JCO.2019.37.15 suppl.4074.

\section{Author Contributions}

All authors made substantial contributions to conception and design, acquisition of data, or analysis and 
interpretation of data; took part in drafting the article or revising it critically for important intellectual content; agreed to submit to the current journal; gave final approval of the version to be published; and agree to be accountable for all aspects of the work.

\section{Funding}

This work was supported by Jiangsu Hengrui Medicine Co., Ltd.

\section{Disclosure}

Xiao Zhang, Linna Wang, Xiaojing Zhang, and Jianjun Zou are employees of Jiangsu Hengrui Medicine Co., Ltd.

The authors declared no other conflicts of interest.

\section{References}

1. El-Serag HB. Hepatocellular carcinoma. N Engl J Med. 2011;365 (12):1118-1127. doi:10.1056/NEJMra1001683

2. Ferlay J, Colombet M, Soerjomataram I, et al. Estimating the global cancer incidence and mortality in 2018: GLOBOCAN sources and methods. Int $J$ Cancer. 2019;144(8):1941-1953. doi:10.1002/ ijc. 31937

3. Allemani C, Weir HK, Carreira H, et al. Global surveillance of cancer survival 1995-2009: analysis of individual data for 25,676,887 patients from 279 population-based registries in 67 countries (CONCORD-2). Lancet. 2015;385(9972):977-1010. doi:10.1016/ S0140-6736(14)62038-9

4. Chen W, Zheng R, Baade PD, et al. Cancer statistics in China, 2015. CA Cancer J Clin. 2016;66(2):115-132. doi:10.3322/caac.21338

5. Cheng AL, Kang YK, Chen Z, et al. Efficacy and safety of sorafenib in patients in the Asia-Pacific region with advanced hepatocellular carcinoma: a phase III randomised, double-blind, placebo-controlled trial. Lancet Oncol. 2009;10(1):25-34. doi:10.1016/S1470-2045(08) 70285-7

6. Llovet JM, Ricci S, Mazzaferro V, et al. Sorafenib in advanced hepatocellular carcinoma. $N$ Engl J Med. 2008;359(4):378-390. doi:10.1056/NEJMoa0708857

7. Kudo M, Finn RS, Qin S, et al. Lenvatinib versus sorafenib in first-line treatment of patients with unresectable hepatocellular carcinoma: a randomised Phase 3 non-inferiority trial. Lancet. 2018;391 (10126):1163-1173. doi:10.1016/S0140-6736(18)30207-1

8. Qin S, Bai Y, Lim HY, et al. Randomized, multicenter, open-label study of oxaliplatin plus fluorouracil/leucovorin versus doxorubicin as palliative chemotherapy in patients with advanced hepatocellular carcinoma from Asia. J Clin Oncol. 2013;31(28):3501-3508. doi:10.1200/JCO.2012.44.5643

9. Qin S, Cheng Y, Liang J, et al. Efficacy and safety of the FOLFOX4 regimen versus doxorubicin in Chinese patients with advanced hepatocellular carcinoma: a subgroup analysis of the EACH study. Oncologist. 2014;19(11):1169-1178. doi:10.1634/theoncologist.2014-0190

10. NCCN clinical practice guidelines in oncology. hepatobiliary cancers. Version 5; 2020. Available from: https://www.ncen.org/professionals/ physician_gls/pdf/hepatobiliary.pdf. Accessed March 02, 2021.

11. Bruix J, Qin S, Merle P, et al. Regorafenib for patients with hepatocellular carcinoma who progressed on sorafenib treatment (RESORCE): a randomised, double-blind, placebo-controlled, phase 3 trial. Lancet. 2017;389(10064):56-66. doi:10.1016/S0140-6736(16) 32453-9
12. Abou-Alfa GK, Meyer T, Cheng AL, et al. Cabozantinib in patients with advanced and progressing hepatocellular carcinoma. $N$ Engl $J$ Med. 2018;379(1):54-63. doi:10.1056/NEJMoa1717002

13. Zhu AX, Kang YK, Yen CJ, et al. Ramucirumab after sorafenib in patients with advanced hepatocellular carcinoma and increased alpha-fetoprotein concentrations (REACH-2): a randomised, double-blind, placebo-controlled, phase 3 trial. Lancet Oncol. 2019;20(2):282-296. doi:10.1016/S1470-2045(18)30937-9

14. El-Khoueiry AB, Sangro B, Yau T, et al. Nivolumab in patients with advanced hepatocellular carcinoma (CheckMate 040): an open-label, non-comparative, Phase 1/2 dose escalation and expansion trial. Lancet. 2017;389(10088):2492-2502. doi:10.1016/S0140-6736(17) 31046-2

15. Yau T, Hsu C, Kim TY, et al. Nivolumab in advanced hepatocellular carcinoma: sorafenib-experienced Asian cohort analysis. J Hepatol. 2019;71(3):543-552. doi:10.1016/j.jhep.2019.05.014

16. Zhu AX, Finn RS, Edeline J, et al. Pembrolizumab in patients with advanced hepatocellular carcinoma previously treated with sorafenib (KEYNOTE-224): a non-randomised, open-label Phase 2 trial. Lancet Oncol. 2018;19(7):940-952. doi:10.1016/S1470-2045(18) 30351-6

17. Qin S, Ren Z, Meng Z, et al. Camrelizumab in patients with previously treated advanced hepatocellular carcinoma: a multicentre, open-label, parallel-group, randomised, phase 2 trial. Lancet Oncol. 2020;21(4):571-580. doi:10.1016/S1470-2045(20)30011-5

18. Yau T, Park JW, Finn RS, et al. LBA38_PR - CheckMate 459: a randomized, multi-center phase III study of nivolumab (NIVO) vs sorafenib (SOR) as first-line (1L) treatment in patients (pts) with advanced hepatocellular carcinoma (aHCC). Ann Oncol. 2019;30: v874-v875. doi:10.1093/annonc/mdz394.029

19. Finn RS, Ryoo BY, Merle P, et al. Pembrolizumab as second-line therapy in patients with advanced hepatocellular carcinoma in KEYNOTE-240: a randomized, double-blind, Phase III Trial. J Clin Oncol. 2020;38(3):193-202. doi:10.1200/JCO.19.01307

20. Tian M, Shi Y, Liu W, Fan J. Immunotherapy of hepatocellular carcinoma: strategies for combinatorial intervention. Sci China Life Sci. 2019;62(9):1138-1143. doi:10.1007/s11427-018-9446-2

21. Chen M, Li L, Li H, et al. Treatment patterns and clinical outcomes for patients with hepatocellular carcinoma from China: a sub-analysis of the Global Hepatocellular Carcinoma BRIDGE Study. $J$ Hepatol. 2017;66(1):S455-S456. doi:10.1016/S0168-8278(17)31291-6

22. Finn RS, Ikeda M, Zhu AX, et al. Phase Ib study of lenvatinib plus pembrolizumab in patients with unresectable hepatocellular carcinoma. J Clin Oncol. 2020;38(26):2960-2970. doi:10.1200/ JCO.20.00808

23. Finn RS, Qin S, Ikeda M, et al. Atezolizumab plus bevacizumab in unresectable hepatocellular carcinoma. $N$ Engl J Med. 2020;382 (20):1894-1905. doi:10.1056/NEJMoa1915745

24. Langer CJ, Gadgeel SM, Borghaei H, et al. Carboplatin and pemetrexed with or without pembrolizumab for advanced, non-squamous non-small-cell lung cancer: a randomised, phase 2 cohort of the open-label KEYNOTE-021 study. Lancet Oncol. 2016;17 (11):1497-1508. doi:10.1016/S1470-2045(16)30498-3

25. Borghaei H, Langer CJ, Gadgeel S, et al. 24-Month Overall Survival from KEYNOTE-021 Cohort G: pemetrexed and carboplatin with or without pembrolizumab as first-line therapy for advanced nonsquamous non-small cell lung cancer. $J$ Thorac Oncol. 2019;14 (1):124-129. doi:10.1016/j.jtho.2018.08.004

26. Paz-Ares L, Luft A, Vicente D, et al. Pembrolizumab plus chemotherapy for squamous non-small-cell lung cancer. $N$ Engl $J$ Med. 2018;379(21):2040-2051. doi:10.1056/NEJMoa1810865

27. Fang W, Yang Y, Ma Y, et al. Camrelizumab (SHR-1210) alone or in combination with gemcitabine plus cisplatin for nasopharyngeal carcinoma: results from two single-arm, phase 1 trials. Lancet Oncol. 2018;19(10):1338-1350. doi:10.1016/S1470-2045(18)30495-9 
28. Lv JW, Li JY, Luo LN, Wang ZX, Chen YP. Comparative safety and efficacy of anti-PD-1 monotherapy, chemotherapy alone, and their combination therapy in advanced nasopharyngeal carcinoma: findings from recent advances in landmark trials. J Immunother Cancer. 2019;7(1):159. doi:10.1186/s40425-019-0636-7

29. Peters S, Kim AW, Solomon B, et al. IMpower030: phase III study evaluating neoadjuvant treatment of resectable stage II-IIIB non-small cell lung cancer (NSCLC) with atezolizumab (atezo) + chemotherapy. Ann Oncol. 2019;30:ii30. doi:10.1093/annonc/ mdz064.014

30. Zhou C, Chen G, Huang Y, et al. OA04.03 A randomized phase 3 study of camrelizumab plus chemotherapy as 1st Line therapy for advanced/metastatic non-squamous non-small cell lung cancer. $J$ Thoracic Oncol. 2019;14(10):S215-S216. doi:10.1016/j.jtho.20 19.08 .425

31. Wang Q, Ju X, Wang J, Fan Y, Ren M, Zhang H. Immunogenic cell death in anticancer chemotherapy and its impact on clinical studies. Cancer Lett. 2018;438:17-23. doi:10.1016/j.canlet.2018.08.028

32. Emens LA, Middleton G. The interplay of immunotherapy and chemotherapy: harnessing potential synergies. Cancer Immunol Res. 2015;3(5):436-443. doi:10.1158/2326-6066.CIR-15-0064

33. Grimaldi A, Cammarata I, Martire C, et al. Combination of chemotherapy and PD-1 blockade induces $\mathrm{T}$ cell responses to tumor non-mutated neoantigens. Commun Biol. 2020;3(1):85. doi:10.1038/ s42003-020-0811-x
34. Jafari S, Lavasanifar A, Hejazi MS, Maleki-Dizaji N, Mesgari M, Molavi O. STAT3 inhibitory stattic enhances immunogenic cell death induced by chemotherapy in cancer cells. Daru. 2020;28(1):159-169. doi: $10.1007 / \mathrm{s} 40199-020-00326-\mathrm{z}$

35. Tu K, Deng H, Kong L, et al. Reshaping tumor immune microenvironment through acidity-responsive nanoparticles featured with CRISPR/Cas9-mediated programmed death-ligand 1 attenuation and chemotherapeutics-induced immunogenic cell death. ACS Appl Mater Interfaces. 2020;12(14):16018-16030. doi:10.1021/acsami.9b23084

36. Chen DS, Mellman I. Elements of cancer immunity and the cancer-immune set point. Nature. 2017;541(7637):321-330. doi:10.1038/nature21349

37. Rebe C, Demontoux L, Pilot T, Ghiringhelli F. Platinum derivatives effects on anticancer immune response. Biomolecules. 2019;10(1). doi: $10.3390 /$ biom 10010013

38. Wang W, Wu L, Zhang J, Wu H, Han E, Guo Q. Chemoimmunotherapy by combining oxaliplatin with immune checkpoint blockades reduced tumor burden in colorectal cancer animal model. Biochem Biophys Res Commun. 2017;487(1):1-7. doi:10.1016/j.bbrc.2016.12.180

39. Wang F, Qin S, Sun X, et al. Reactive cutaneous capillary endothelial proliferation in advanced hepatocellular carcinoma patients treated with camrelizumab: data derived from a multicenter phase 2 trial. J Hematol Oncol. 2020;13(1):47. doi:10.1186/s13045-020-00886-2

\section{Publish your work in this journal}

Drug Design, Development and Therapy is an international, peerreviewed open-access journal that spans the spectrum of drug design and development through to clinical applications. Clinical outcomes, patient safety, and programs for the development and effective, safe, and sustained use of medicines are a feature of the journal, which has also been accepted for indexing on PubMed Central. The manuscript management system is completely online and includes a very quick and fair peer-review system, which is all easy to use. Visit http://www. dovepress.com/testimonials.php to read real quotes from published authors. 